\title{
Painlevé analysis and exact solutions for the coupled Burgers system
}

\author{
P. Barrera ${ }^{1} \&$ T. Brugarino ${ }^{2}$ \\ ${ }^{1}$ Dipartimento di Meccanica, Università di Palermo, \\ Facoltà d'Ingegneria, Palermo, Italia \\ ${ }^{2}$ Dipartimento di Metodi e Modelli Matematici, Università di Palermo, \\ Facoltà d'Ingegneria, Palermo, Italia
}

\begin{abstract}
We perform the Painlevé test to a system of two coupled Burgers-type equations which fails to satisfy the Painlevé test. In order to obtain a class of solutions, we use a slightly modified version of the test. These solutions are expressed in terms of the Airy functions. We also give the travelling wave solutions, expressed in terms of the trigonometric and hyperbolic functions.
\end{abstract}

\section{Introduction}

The nonlinear diffusion-convection equations

$$
\left\{\begin{aligned}
& u_{t}(x, t)=u_{x x}(x, t)+\mu u(x, t) u_{x}(x, t)+\lambda_{11} v(x, t) u_{x}(x, t) \\
&+ \lambda_{12} u(x, t) v_{x}(x, t) \\
& v_{t}(x, t)=v_{x x}(x, t)+\nu v(x, t) v_{x}(x, t)+\lambda_{21} v(x, t) u_{x}(x, t) \\
&+\lambda_{22} u(x, t) v_{x}(x, t)
\end{aligned}\right.
$$

have a lot of applications in physics, chemistry and biology [1-3], particularly in the study of porous media [4], in polydispersive sedimentation [5], in dynamic of growing interfaces [6] and in the study of integrable coupled Burgers-type equations [7], [8].

The paper is organized as follows: in sect. 2 we show that system (1), for arbitrary coefficients, is not integrable in Painlevé sense; in sect. $\mathbf{3}$ a slightly modified version of the truncated Painlevé test is used to obtain analytic solutions for particular values of the coefficients; in sect. 4 we determine some exact solutions of 
the system with the aid of the Airy, the trigonometric and the hyperbolic functions. The last section is devoted to brief conclusions.

\section{Painlevé analysis}

It is possible to study the complete integrability of a system of partial or ordinary differential equations by using the so-called Painlevé test [9], [10]. The Painlevé test for the integrability of a system of partial differential equations

$$
\mathbf{u}_{t}=\mathbf{K}(x, t, \mathbf{u})
$$

presented by Weiss-Tabor-Carnevale [9] involves seeking solutions of eq. (2) as expansion of the form of a Laurent series:

$$
\mathbf{u}(x, t)=\phi(x, t)^{\mathbf{a}} \sum_{j=0}^{\infty} \mathbf{u}_{j} \phi(x, t)^{j}
$$

where each vector $\mathbf{u}_{j}$ is a function of $x$ and $t$, and the $\phi(t, x)=0$ defines an arbitrary noncharacteristic movable singular manifold. The Painlevé method consists of the following main steps:

(i) determination of the leading behaviour;

(ii) identification of the powers at which arbitrary functions can enter in to the Laurent series, called resonances;

(iii) verifying that at resonance values a sufficient number of arbitrary functions exists without the introduction of the movable critical points.

First, we show that the system (1) fails to satisfy the Painlevé test. We assume that the solutions of the system (1) take the form:

$$
\begin{aligned}
& u(x, t)=\phi(x, t)^{a} \sum_{j=0}^{\infty} u(x, t)_{j} \phi(x, t)^{j} \\
& v(x, t)=\phi(x, t)^{b} \sum_{j=0}^{\infty} v(x, t)_{j} \phi(x, t)^{j}
\end{aligned}
$$

where $a$ and $b$ are negative integers. By leading order analysis, we find that $a=$ $b=-1$. Inserting in system (1) the expansions (3) and (4), we obtain

$$
\left\{\begin{array}{c}
\mu u_{0}^{2} \phi_{x}+\lambda_{11} u_{0} v_{0} \phi_{x}+\lambda_{12} u_{0} v_{0} \phi_{x}-2 u_{0} \phi_{x}^{2}=0 \\
\lambda_{21} u_{0} v_{0} \phi_{x}+\lambda_{22} u_{0} v_{0} \phi_{x}+\nu v_{0}^{2} \phi_{x}-2 v_{0} \phi_{x}^{2}=0
\end{array}\right.
$$

Solving eqns. (5), we have

$$
\left\{\begin{array}{l}
u_{0}=\frac{2\left(\lambda_{11}+\lambda_{12}-\nu\right) \phi_{x}}{\lambda_{11}\left(\lambda_{21}+\lambda_{22}\right)+\lambda_{12}\left(\lambda_{21}+\lambda_{22}\right)-\mu \nu} \\
v_{0}=\frac{2\left(\lambda_{21}+\lambda_{22}-\mu\right) \phi_{x}}{\lambda_{11}\left(\lambda_{21}+\lambda_{22}\right)+\lambda_{12}\left(\lambda_{21}+\lambda_{22}\right)-\mu \nu}
\end{array}\right.
$$


We take the meaningful values of $u_{0}$ and $v_{0}$. Substituting the Laurent series (3) in eqns. (1), collecting the coefficients of $\phi_{x}^{r-3}$ and utilizing eqns. (6), we finally obtain the resonance values:

$$
\begin{gathered}
r_{1}=-1, \quad r_{2}=2 \\
r_{3,4}=3 \lambda_{11} \lambda_{21}+5 \lambda_{12} \lambda_{21}+\lambda_{11} \lambda_{22}+3 \lambda_{12} \lambda_{22}-2 \lambda_{12} \mu-2 \lambda_{21} \nu-\mu \nu \\
\frac{ \pm \sqrt{\left(\lambda_{11}\left(3 \lambda_{21}+\lambda_{22}\right)+\lambda_{12}\left(5 \lambda_{21}+3 \lambda_{22}-2 \mu\right)-\left(2 \lambda_{21}+\mu\right) \nu\right)^{2}-}}{8\left(\lambda_{21}+\lambda_{22}-\mu\right)\left(\lambda_{11}+\lambda_{12}-\nu\right)\left(\left(\lambda_{11}+\lambda_{12}\right)\left(\lambda_{21}+\lambda_{22}\right)-\mu \nu\right)} \times \\
\left(2\left(\lambda_{11}+\lambda_{12}\right)\left(\lambda_{21}+\lambda_{22}\right)-2 \mu \nu\right)^{-1}
\end{gathered}
$$

Because the values of $r_{3,4}$ are not integer, for any value of $\lambda_{h k}$, the system (1) does not possess the Painlevé property.

\section{Truncated Painlevé analysis}

Now we use the truncated version of the Painlevé test to look for solutions of a system of differential equations [9] and [11].

The invariant formalism of truncated Painlevé [10], [12] implies looking for a solutions of eqns. (1) as:

$$
\left\{\begin{array}{l}
u=U_{1} \omega+U_{0} \\
v=V_{1} \omega+V_{0}
\end{array}\right.
$$

where the $\omega=\Psi_{x} / \Psi$.

The variable $\omega$ satisfies the Riccati equations

$$
\begin{gathered}
\omega_{x}=-\omega^{2}-\frac{S}{2} \\
\omega_{t}=C \omega^{2}-\omega C_{x}+\frac{C S+C_{x x}}{2}
\end{gathered}
$$

and the variable $\Psi$ satisfies the linear equations

$$
\begin{gathered}
\Psi_{x x}=-\frac{1}{2} S \Psi \\
\Psi_{t}=\frac{1}{2} C_{x} \Psi-C \Psi_{x}
\end{gathered}
$$

The coefficients are related by the cross-derivative condition

$$
S_{t}+2 S C_{x}+C S_{x}+C_{x x x}=0
$$

where $S$, the Schwarzian derivative of $\phi$, and $C$ are defined by:

$$
S=\frac{\phi_{x x x}}{\phi_{x}}-\frac{3}{2}\left(\frac{\phi_{x x}}{\phi_{x}}\right)^{3} \quad C=-\frac{\phi_{t}}{\phi_{x}}
$$

Substituting eqns. (7) in eqns. (1), then for eqns. (7) to be a solution, we must have: 
For $j=1$ :

$$
\begin{aligned}
& U_{1}=\frac{2\left(\lambda_{11}+\lambda_{12}-\nu\right)}{\left(\lambda_{11}+\lambda_{12}\right)\left(\lambda_{21}+\lambda_{22}\right)-\mu \nu} \\
& V_{1}=\frac{2\left(\lambda_{21}+\lambda_{22}-\mu\right)}{\left(\lambda_{11}+\lambda_{12}\right)\left(\lambda_{21}+\lambda_{22}\right)-\mu \nu}
\end{aligned}
$$

for $j=2$ :

$$
\begin{aligned}
& U_{0}=-\frac{\left(\lambda_{11}+\lambda_{12}-\nu\right) C}{\left(\lambda_{11}+\lambda_{12}\right)\left(\lambda_{21}+\lambda_{22}\right)-\mu \nu} \\
& V_{0}=-\frac{\left(\lambda_{21}+\lambda_{22}-\mu\right) C}{\left(\lambda_{11}+\lambda_{12}\right)\left(\lambda_{21}+\lambda_{22}\right)-\mu \nu}
\end{aligned}
$$

therefore

$$
\begin{aligned}
& u=\frac{\lambda_{11}+\lambda_{12}-\nu}{\left(\lambda_{11}+\lambda_{12}\right)\left(\lambda_{21}+\lambda_{22}\right)-\mu \nu}(2 \omega-C) \\
& v=\frac{\lambda_{21}+\lambda_{22}-\nu}{\left(\lambda_{11}+\lambda_{12}\right)\left(\lambda_{21}+\lambda_{22}\right)-\mu \nu}(2 \omega-C)
\end{aligned}
$$

and, for $j=3$ :

$$
C_{t}+C C_{x}-S_{x}-2 C_{x x}=0
$$

and, of course, the cross-derivative condition

$$
S_{t}+2 S C_{x}+C S_{x}+C_{x x x}=0
$$

\section{Exact solutions}

Now we able to obtain solutions of system (1), by solving eqns. (9), (10) and (8). A possible set of solutions of eqns. (9) and (10) is:

$$
\begin{gathered}
C=a t+c \\
S=a x-\frac{a^{2} t^{2}}{2}-a c t+d
\end{gathered}
$$

while eqn. (8):

$$
\Psi_{x x}+\left(a x-\frac{a^{2} t^{2}}{2}-a c t+d\right) \frac{\Psi}{2}=0
$$

is now an ordinary differential equation of Airy type [13], the variable $t$ acting as a parameter. We consider three cases. 


\section{1 $a \neq 0$}

The Airy equation has the following general solution

$$
\begin{aligned}
\Psi= & k_{1} \operatorname{Ai}\left(\sqrt[3]{(2 / a)^{2}}\left(\frac{a^{2} t^{2}+2 a c t-2 d}{4}-\frac{a x}{2}\right)\right)+ \\
& k_{2} \operatorname{Bi}\left(\sqrt[3]{(2 / a)^{2}}\left(\frac{a^{2} t^{2}+2 a c t-2 d}{4}-\frac{a x}{2}\right)\right)
\end{aligned}
$$

therefore, the solutions of system (1) are:

$$
\begin{aligned}
& u=\frac{\lambda_{11}+\lambda_{12}-\nu}{\left(\lambda_{11}+\lambda_{12}\right)\left(\lambda_{21}+\lambda_{22}\right)-\mu \nu}(a t+c+ \\
& \left.\sqrt[3]{4 a} \frac{\operatorname{Ai}^{\prime}\left(\sqrt[3]{4 a}\left(\frac{a t^{2}}{4}+\frac{c t}{2}-\frac{d}{2 a}-\frac{x}{2}\right)\right)+k \mathrm{Bi}^{\prime}\left(\sqrt[3]{4 a}\left(\frac{a t^{2}}{4}+\frac{c t}{2}-\frac{d}{2 a}-\frac{x}{2}\right)\right)}{\operatorname{Ai}\left(\sqrt[3]{4 a}\left(\frac{a t^{2}}{2}+\frac{c t}{2}-\frac{d}{2 a}-\frac{x}{2}\right)\right)+k \operatorname{Bi}\left(\sqrt[3]{4 a}\left(\frac{a t^{2}}{2}+\frac{c t}{2}-\frac{d}{2 a}-\frac{x}{2}\right)\right)}\right) \\
& v=\frac{\lambda_{22}+\lambda_{21}-\mu}{\left(\lambda_{11}+\lambda_{12}\right)\left(\lambda_{21}+\lambda_{22}\right)-\mu \nu}(a t+c+ \\
& \left.\sqrt[3]{4 a} \frac{\operatorname{Ai}^{\prime}\left(\sqrt[3]{4 a}\left(\frac{a t^{2}}{4}+\frac{c t}{2}-\frac{d}{2 a}-\frac{x}{2}\right)\right)+k \operatorname{Bi}^{\prime}\left(\sqrt[3]{4 a}\left(\frac{a t^{2}}{4}+\frac{c t}{2}-\frac{d}{2 a}-\frac{x}{2}\right)\right)}{\operatorname{Ai}\left(\sqrt[3]{4 a}\left(\frac{a t^{2}}{2}+\frac{c t}{2}-\frac{d}{2 a}-\frac{x}{2}\right)\right)+k \operatorname{Bi}^{2}\left(\sqrt[3]{4 a}\left(\frac{a t^{2}}{2}+\frac{c t}{2}-\frac{d}{2 a}-\frac{x}{2}\right)\right)}\right)
\end{aligned}
$$

where $\mathrm{Ai}$ and $\mathrm{Bi}$ are the Airy functions, the prime denotes the derivative with respect to the argument, and $k$ is an arbitrary constant.

$$
4.2 a=0, d=-\delta(\delta>0)
$$

The eqn. (12) has the general solution expressed in terms of the hyperbolic functions, so that the solutions of system (1) are:

$$
\begin{gathered}
u=\frac{\lambda_{11}+\lambda_{12}-\nu}{\left(\lambda_{11}+\lambda_{12}\right)\left(\lambda_{21}+\lambda_{22}\right)-\mu \nu} \times \\
\frac{c+\sqrt{2 \delta}+k(c-\sqrt{2 \delta})(\cosh \sqrt{2 \delta}(x-c t)+\sinh \sqrt{2 \delta}(x-c t))}{1+k(\cosh \sqrt{2 \delta}(x-c t)+\sinh \sqrt{2 \delta}(x-c t))} \\
v=\frac{\lambda_{22}+\lambda_{21}-\mu}{\left(\lambda_{11}+\lambda_{12}\right)\left(\lambda_{21}+\lambda_{22}\right)-\mu \nu} \times \\
\frac{c+\sqrt{2 \delta}+k(c-\sqrt{2 \delta})(\cosh \sqrt{2 \delta}(x-c t)+\sinh \sqrt{2 \delta}(x-c t))}{1+k(\cosh \sqrt{2 \delta}(x-c t)+\sinh \sqrt{2 \delta}(x-c t))}
\end{gathered}
$$


$4.3 a=0, d=\delta(\delta>0)$

In this case, analogously, eqn. (12) has the general solution expressed in terms of the trigonometric functions, and the solutions of system (1) are:

$$
\begin{gathered}
u=\frac{\lambda_{11}+\lambda_{12}-\nu}{\left(\lambda_{11}+\lambda_{12}\right)\left(\lambda_{21}+\lambda_{22}\right)-\mu \nu} \times \\
\frac{c-\sqrt{2 \delta} k+(c k+\sqrt{2 \delta}) \tan ((x-c t) \sqrt{\delta / 2})}{1+k \tan ((x-c t) \sqrt{\delta / 2})} \\
v=\frac{\lambda_{22}+\lambda_{21}-\mu}{1-\left(\lambda_{11}+\lambda_{12}\right)\left(\lambda_{21}+\lambda_{22}\right)-\mu \nu} \times \\
\frac{c-\sqrt{2 \delta} k+(c k+\sqrt{2 \delta}) \tan ((x-c t) \sqrt{\delta / 2})}{1+k \tan ((x-c t) \sqrt{\delta / 2})}
\end{gathered}
$$

\section{Conclusions}

In this note we obtain for the system (1), in addition to the usual travelling wave solutions corresponding to the constant values of the invariant $S$ and $C$, another type of interesting analytic solutions, related to Airy functions, where $S$ and $C$ are not constant.

\section{References}

[1] B. H. Gilding, R. K. Kersner, The characterization of reaction-convectiondiffusion processes by travelling waves, J. Diff. Eqns. 124 (1996) 27.

[2] M. P. Edwards, P. Broadbridge, Exact transient solutions to nonlinear diffusion-convection equations in higher dimensions, J. Phys. A 27 (1994) 5455.

[3] P. Barrera, T. Brugarino, Traveling waves of two coupled nonlinear diffusion convection equations, $14^{\text {th }}$ Symposium Transport and Air Pollution II (2005) 9, Graz.

[4] A. Klute, Soil Sci. 73 (1952) 105.

[5] S. E. Esipov, Coupled Burgers equations: A model of polydispersive sedimentation, Phys. Rev. 52 (1995) 3711.

[6] M. Kardar, G. Parisi, Y. C. Zhang, Dynamic scaling of growing interfaces, Phys. Rev. Lett. 56 (1986) 889.

[7] M. V. Fursov, On integrable coupled Burgers-type equations, Physics Lett. A 272 (2000) 57.

[8] L. Zeng-Ju, C. Li-Li, L. Sen-Yue, Painlevé property, symmetries and symmetry reductions of the coupled Burgers system, Chinese Phys. 14 (2005) 1486. 
[9] J. Weiss, M. Tabor, G. Carnevale, The Painlevé Property for Partial Differential Equations, J. Math. Phys. 24 (1983) 522.

[10] R. Conte, Universal Invariance Properties of Painlevé Analysis and Bäcklund Transformation in Nonlinear Partial Differential Equations, Physics Lett. A 134 (1988) 100.

[11] P. Barrera, T. Brugarino, L. Pignato, Analytic solutions of two coupled reaction-diffusion equations, Air Pollution XI (2003) 135, Catania.

[12] T. Brugarino, Exact Solutions of two Coupled Burgers Equations, Proceedings SIMAI (2002), Cagliari.

[13] M. Abramowitz, I. A. Stegun, Handbook of Mathematical functions, (1972), Dover. 